\title{
Mixed Electrolytes of Organic Solvents and Ionic Liquid for Rechargeable Lithium-Ion Batteries
}

\author{
Ji-Ae Choi, ${ }^{\dagger}$ Eun-Gi Shim, ${ }^{\dagger}$ Bruno Scrosati, ${ }^{\dagger, \S}$ and Dong-Won Kim ${ }^{\dagger, *}$ \\ ${ }^{\dagger}$ Department of Chemical Engineering, Hanyang University, Seungdong-Gu, Seoul 133-791, Korea \\ *E-mail: dongwonkim@hanyang.ac.kr \\ ${ }^{\ddagger}$ Electrolyte Development Team, Techno SemiChem Co. Ltd, Yongin-si, Gyeonggi-do 446-599, Korea \\ ${ }^{\S}$ Department of Chemistry, University of Rome "La Sapienza", Piazzale Aldo Moro 5, 00185 Rome, Italy \\ Received May 26, 2010, Accepted September 6, 2010
}

\begin{abstract}
Mixed electrolytes formed by the combination of 1-butyl-1-methylpyrrolidinium bis(trifluoromethanesulfonyl) imide (BMP-TFSI) ionic liquid and standard liquid electrolyte are prepared and characterized. Linear sweep voltammetry measurements demonstrate that these mixed systems exhibit a wide electrochemical stability window, allowing them to be suitable electrolyte for carbonaceous anode-based lithium-ion batteries. Lithium-ion cells composed of graphite anode and $\mathrm{LiCoO}_{2}$ cathode are assembled using the mixed electrolytes, and their cycling performances are evaluated. The cell containing proper content of BMP-TFSI shows good cycling performance comparable to that of a cell assembled with organic electrolyte. The presence of BMP-TFSI in the mixed electrolyte contributes to the reduction of the flammability of electrolyte solution and the improvement of the thermal stability of charged $\mathrm{Li}_{1-\mathrm{x}} \mathrm{CoO}_{2}$ in the electrolyte solution.
\end{abstract}

Key Words: Cycling performance, Ionic liquid, Lithium-ion battery, Mixed electrolyte, Thermal stability

\section{Introduction}

Lithium-ion batteries have rapidly become the dominant power source for portable electronic devices, power tools and electric vehicles due to their high energy density and long cycle life. However, safety issues surrounding these batteries must be addressed before they can be widely utilized in large-scale cells, such as electric vehicles and energy storage application. The highly flammable organic solvents that are contained in common liquid electrolytes can be the major cause of fire and explosion when short circuiting or local overheating do accidently occur. There is therefore a quest for non-flammable electrolyte systems to replace the organic solutions presently used in lithium-ion batteries. In this respect, large attention is devoted to ionic liquids (ILs), i.e. room temperature molten salts, which recognized as safe electrolyte systems. ${ }^{1-3}$ Indeed, ILs have a series of attractive properties, such as negligible vapor pressure, low flammability, wide electrochemical stability, high ionic conductivity and high thermal stability. Among the various ionic liquid systems, those based on pyrrolidinium cations have been considered to be promising for battery application, due to their reasonably wide electrochemical stability and high ionic conductivity. ${ }^{4,5}$ Accordingly, several electrochemical investigations of pyrrolidinium-based ILs have been reported in the literature. ${ }^{4-10}$ However, to our knowledge, most of these studies have focused on batteries using non-carbonaceous anodes. Probably this lack of attention derived from the occurrence of undesirable irreversible reactions between the carbon anode and the ionic liquid electrolyte. Moreover, most of the relevant IL systems are still very expensive. Thus, the use of IL as additive to standard liquid electrolyte may be beneficial in consideration of reduction of cost.

With the goal of developing the cost-effective IL-based electrolytes for applications in lithium-ion batteries with enhanced safety, the mixed electrolytes compatible for graphitized anode and $\mathrm{LiCoO}_{2}$ cathode have been prepared and characterized. The mixed electrolytes were composed of 1-butyl-1-methylpyrrolidinium bis(trifluoromethanesulfonyl)imide (BMPTFSI) and organic solution. With these mixed electrolytes, we assembled lithium-ion cells and their cycling performances were evaluated. The influence of BMP-TFSI on the flammability of the electrolyte and the thermal stability of the cathode material was also investigated.

\section{Experimental}

Preparation of the mixed electrolytes. BMP-TFSI was purchased from Chem Tech Research Incorporation and used after drying under a vacuum at $100{ }^{\circ} \mathrm{C}$ for $24 \mathrm{hr}$. The water content in BMP-TFSI after drying was determined to be $8 \mathrm{ppm}$ by Karl Fisher titration. Its ionic conductivity was $2.4 \mathrm{mS} \mathrm{cm}^{-1}$ at room temperature. The mixed electrolyte was prepared by adding predetermined amounts of BMP-TFSI to an organic electrolyte. $\mathrm{LiPF}_{6}(1.0 \mathrm{M})$ in EC/DEC (3:7 by volume) containing 1.0 wt \% VC (Techno Semichem Co., Ltd.) was the organic electrolyte of choice. The content of the IL in the mixed electrolyte ranged from 0 to $30 \mathrm{wt} \%$. A porous poly(vinylidene fluoride-co-hexafluoropropylene)-coated polyethylene separator was prepared according to the procedure reported previously. ${ }^{11}$ The polymer-coated separator could be easily wet by the mixed electrolyte solution and retained the electrolyte solution well.

Electrode preparation and cell assembly. The graphite anode was prepared by coating a $N$-methyl pyrrolidone (NMP)-based slurry of mesocarbon microbeads (MCMB, Osaka Gas), poly (vinylidene fluoride) (PVdF) and super-P carbon (MMM Co.) on a copper foil. The cathode contained $\mathrm{LiCoO}_{2}$ (Japan Che- 
mical), along with the same binder and super-P carbon, which were cast on an aluminum foil. The thickness of the electrodes after roll pressing ranged from 55 to $70 \mu \mathrm{m}$, and their active mass loading corresponded to a capacity of $2.9 \mathrm{mAh} \mathrm{cm}^{-2}$. The cell, composed of a carbon anode, a polymer-coated separator wet by the mixed electrolyte and $\mathrm{LiCoO}_{2}$ cathode was assembled in a dry box filled with argon gas, enclosed in a pouch bag and finally vacuum-sealed.

Measurements. Linear sweep voltammetry (LSV) was performed to investigate the electrochemical stability of the mixed electrolyte on a stainless steel working electrode, with counter and reference electrodes of lithium metal, at a scanning rate of $1.0 \mathrm{mVs}^{-1}$. The self-extinguishing time (SET) was measured to quantify the flammability of the mixed electrolyte, as described in previous literature. ${ }^{12,13}$ It was obtained by igniting the pre-weighed electrolytes soaked in an inert glass-fiber wick $(3 \times$ $3 \mathrm{~cm}^{2}$ ), followed by recording the time it took for the flame to extinguish. The measurements were repeated at least five times to get reproducible SET values. AC impedance measurements were performed using an impedance analyzer over the frequency range of $1 \mathrm{mHz}-100 \mathrm{kHz}$ with an amplitude of $10 \mathrm{mV}$. Charge and discharge cycling tests of the lithium-ion cells were conducted at a current density of $1.45 \mathrm{~mA} \mathrm{~cm}^{-2}(0.5 \mathrm{C}$ rate $)$ over a voltage range of $3.0-4.2 \mathrm{~V}$ with battery test equipment. For differential scanning calorimetry (DSC) experiments, the cells were fully charged to $4.2 \mathrm{~V}$ and disassembled in the dry box. Approximately $5 \mathrm{mg}$ of the cathode scraped from the current collector was hermetically sealed in a stainless steel pan, and measurements were taken at a heating rate of $1^{\circ} \mathrm{C} \mathrm{min}^{-1}$.

\section{Results and Discussion}

Figure 1 shows the cathodic and anodic LSV curves for electrolytes at various compositions, namely, i) the pure organic electrolyte (0 wt \% BMP-TFSI), ii) the mixed electrolyte (10 $30 \mathrm{wt} \% \mathrm{BMP}-\mathrm{TFSI})$ and iii) the pure ionic liquid electrolyte (100 wt \% BMP-TFSI). It can be seen in Figure 1(a) that the cathodic current starts to increase around $1.5 \mathrm{~V} v s \mathrm{Li} / \mathrm{Li}^{+}$in the case of the pure ionic liquid electrolyte, which corresponds to the reductive decomposition of the 1-butyl-1-methylpyrrolidinium cation. This value of cathodic decomposition is in agreement with that observed in LiTFSI and 1-butyl-1-ethylpyrrolidinium bis(trifluoromethanesulfonyl)imide solution by Scrosati and co-workers. ${ }^{5}$ For the mixed electrolytes, small and broad reduction peaks are observed in the potential range between 0.5 and $1.8 \mathrm{~V}$. They can be attributed to the reductive decomposition of organic solvents, such as EC and VC, which result in the formation of a solid electrolyte interphase (SEI) film. Extensive investigations of electrolyte systems have shown that EC and VC easily decompose and leave highly stable SEI film on the electrode surface at potentials more positive than the main solvent decomposes. ${ }^{14-21}$ This SEI film prevents the reductive decomposition of the BMP cation in the IL, this kinetically extending the cathodic stability to $0 \mathrm{~V} v s \mathrm{Li} / \mathrm{Li}^{+}$. The current associated with the lithium deposition is in fact significantly enlarged, suggesting that the addition of the organic solution may indeed allow the ongoing of electrochemical processes down to $0 \mathrm{~V} v s \mathrm{Li} / \mathrm{Li}^{+}$. In the anodic scan shown in Figure 1(b), (a)

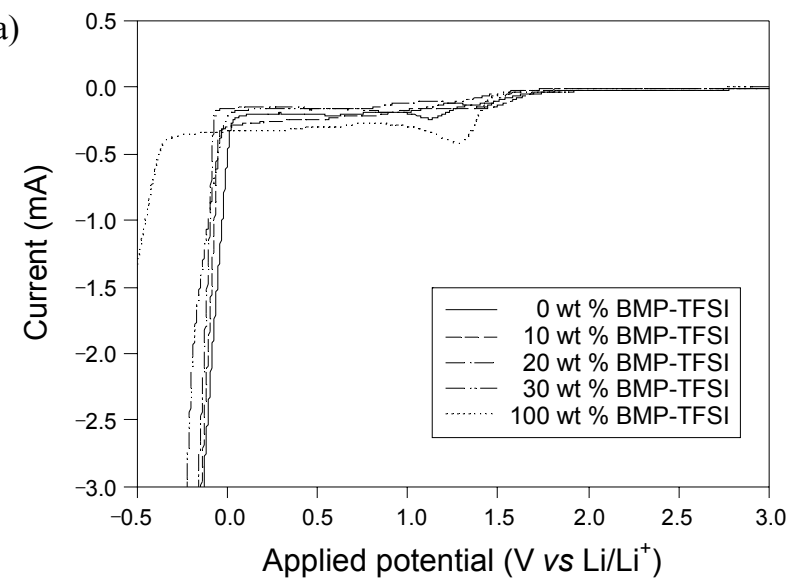

(b)

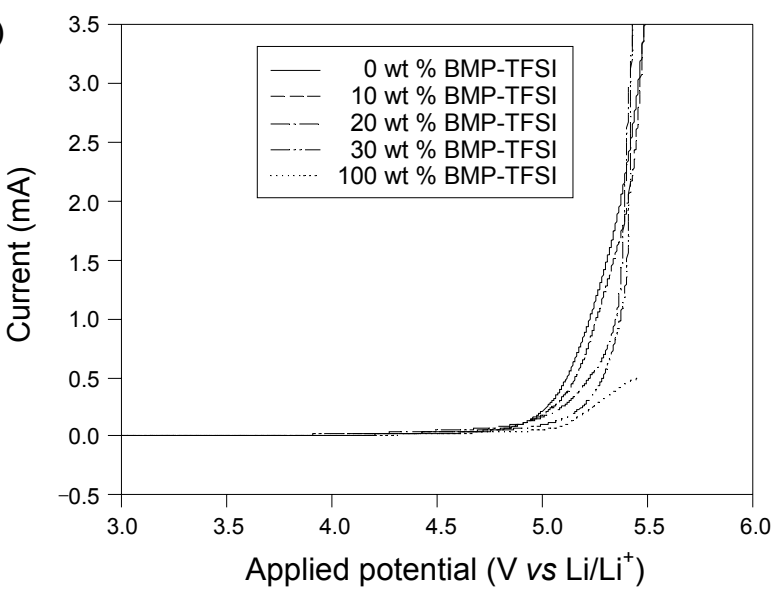

Figure 1. Linear sweep voltammograms of the mixed electrolytes composed of BMP-TFSI and organic solution: (a) cathodic scan, and (b) anodic scan. Room temperature. Scan rate: $1.0 \mathrm{mVs}^{-1}$.

the decomposition potential slightly increases with increasing BMP-TFSI content in the mixed electrolytes, demonstrating that the addition of BMP-TFSI to the organic solution can increase the anodic stability of the electrolyte. In view of the above results, it is expected that the mixed electrolyte considered in this study has an electrochemical stability suitable for allowing the electrochemical operation of graphite/ $\mathrm{LiCoO}_{2}$ cells.

Cycling performances of lithium-ion cells prepared with mixed electrolyte were evaluated at $25^{\circ} \mathrm{C}$. After three preconditioning cycles at low current rate $\left(0.1 \mathrm{C}\right.$ rate, $\left.0.29 \mathrm{~mA} \mathrm{~cm}^{-2}\right)$, the cell was charged at a constant current $(\mathrm{CC})$ of $1.45 \mathrm{~mA} \mathrm{~cm}^{-2}$ $(0.5 \mathrm{C}$ rate) up to the target voltage of $4.2 \mathrm{~V}$. This was followed by a constant voltage $(\mathrm{CV})$ charge with a decline in the current until a final current of $20 \%$ of the charging current was reached. The cell was then discharged down to a cut-off voltage of $3.0 \mathrm{~V}$ at the same current density $(0.5 \mathrm{C}$ rate). Figure 2 shows the charge-discharge curves of the 1st, 10th, 50th and 100th cycle of a representative lithium-ion cell assembled with the mixed electrolyte containing $20 \mathrm{wt} \%$ BMP-TFSI. The charge and discharge curves are almost identical to those for the cell using a pure organic electrolyte, indicating that the basic electrochemical process, involving the cycling of lithium ions between anode and cathode, holds also when the ionic liquid is added into the organic electrolyte. Therefore, one can reasonably assume that 


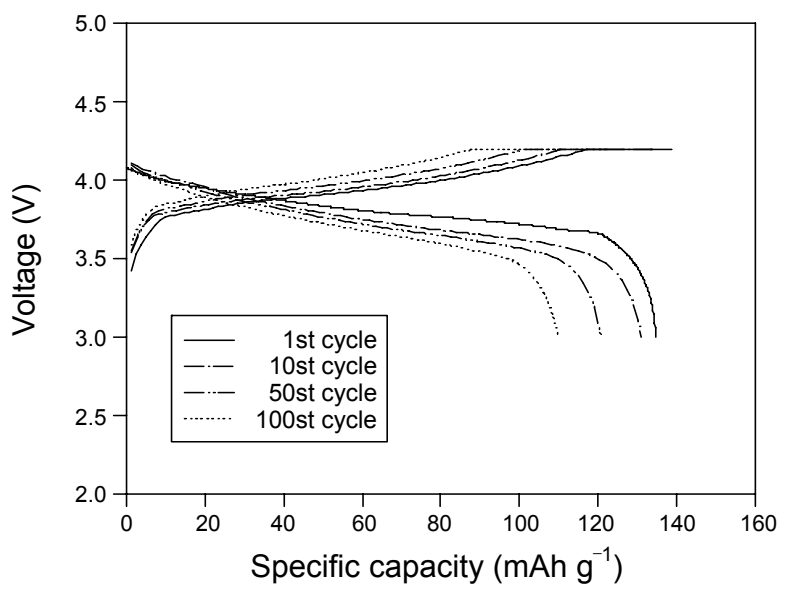

Figure 2. Charge and discharge curves of the lithium-ion cell assembled with mixed electrolyte containing $20 \mathrm{wt} \%$ BMP-TFSI (Room temperature, $0.5 \mathrm{C} \mathrm{CC}$ and $\mathrm{CV}$ charge, $0.5 \mathrm{C} \mathrm{CC}$ discharge, cut-off voltage : $3.0-4.2 \mathrm{~V}$ ).

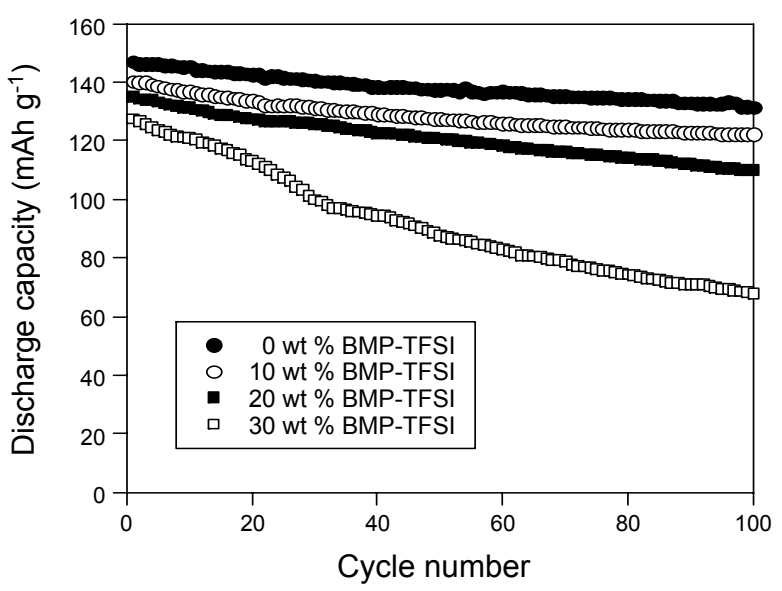

Figure 3. Discharge capacities as a function of cycle number for lithium-ion cells prepared with mixed electrolytes containing different content of BMP-TFSI (Room temperature, $0.5 \mathrm{C} \mathrm{CC}$ and CV charge, 0.5 C CC discharge, cut-off voltage range: $3.0-4.2 \mathrm{~V}$ ).

also in the case of our mixed-electrolyte cell, the graphite anode is covered by a good SEI film that prevents IL decomposition, this finally allowing an efficient and reproducible cycling response. This SEI film is induced by the coexistence of organic solvents, such as EC and VC, as previously outlined when discussing the LSV results of Figure 1. Obviously, the SEI helps to prevent both the reductive decomposition of BMP-TFSI and the co-insertion of the BMP cation into the graphite layer, which are critical issues for most of the IL-based electrolytes. Indeed, the formation of SEI prior to reduction of IL cations (ammonium or imidazolium) in the presence of EC and $\mathrm{VC}$ was evidenced by previous works. ${ }^{21-23}$ The cell shown in Figure 2 exhibited a first discharge capacity of $135 \mathrm{mAh} \mathrm{g}^{-1}$ based on the $\mathrm{LiCoO}_{2}$ active cathode material. The discharge capacity of the cell declined to $110 \mathrm{mAh} \mathrm{g}^{-1}$ after 100 cycles, while the coulombic efficiency steadily increased with cycle number. Figure 3 shows the effect of the BMP-TFSI content in the electrolyte on the discharge capacities of the cells, as a function of cycle (a)

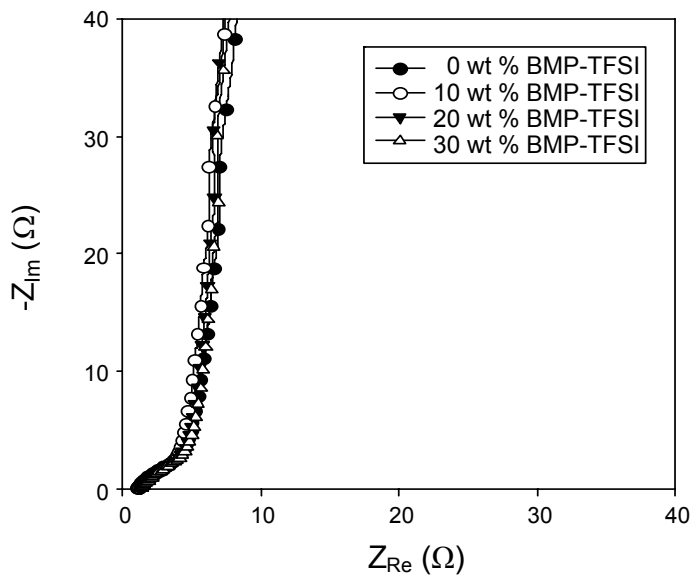

(b)

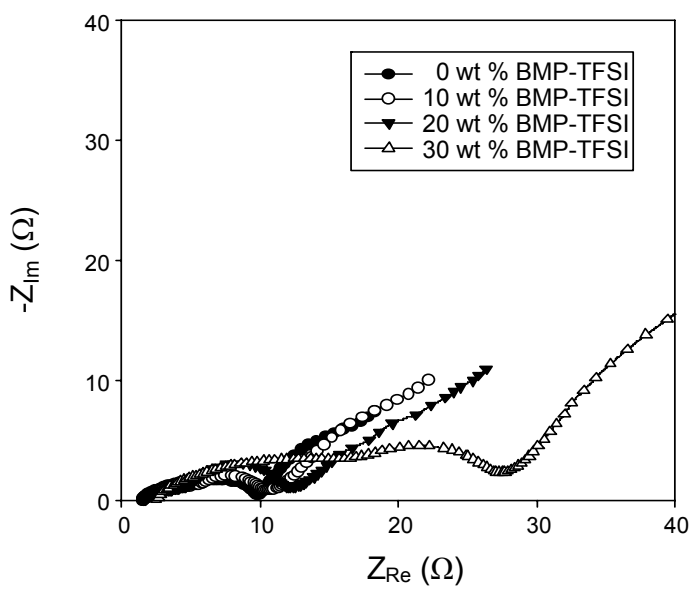

Figure 4. AC impedance spectra (a) before and (b) after 100 cycles of the lithium-ion cells assembled with mixed electrolytes containing different content of BMP-TFSI.

number. Clearly, the initial discharge capacity slightly decreases with increasing BMP-TFSI content. It can be explained considering that the addition of BMP-TFSI into the organic solution causes an increase in viscosity, which results in an increase of the resistance for ion migration in the electrolyte and charge transfer in the electrode. Figure 3 also reveals that the capacity retention of the cell is dependent on the content of BMP-TFSI in the mixed electrolyte. Especially, when the content of BMPTFSI is $30 \mathrm{wt} \%$, significant capacity fading during cycling is observed. Even though the organic solvents can induce SEI films that protect undesirable irreversible reactions at the carbon/ BMP-TFSI interface, this protection many not be totally assured, especially for high-BMP-TFSI content. The occurrence of these reactions may explain the observed gradual decrease of discharge capacity. From these result, it can be concluded that the content of BMP-TFSI in the mixed electrolyte should be kept less than $30 \mathrm{wt} \%$ for efficient cell operation.

In order to investigate the effect of the BMP-TFSI content on the impedance behavior of the cell, the ac impedance of the cells before and after the repeated cycles ( 100 cycles) was measured, and the results are shown in Figure 4. Figure 4(a) was obtained at the open circuit potential before cycling, and Figure 4(b) was measured at the charged state after 100 cycles. The pristine cells under open circuit voltage exhibited almost iden- 


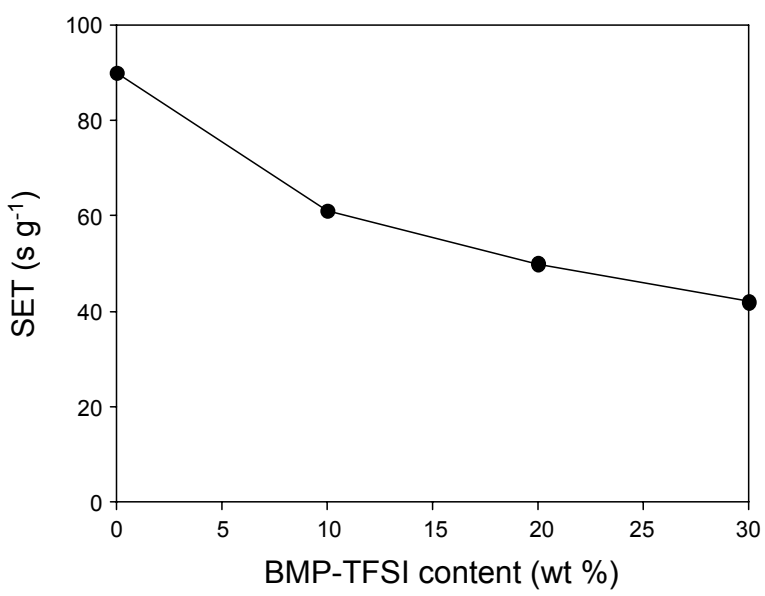

Figure 5. SET values of the mixed electrolytes as a function of the content of BMP-TFSI.

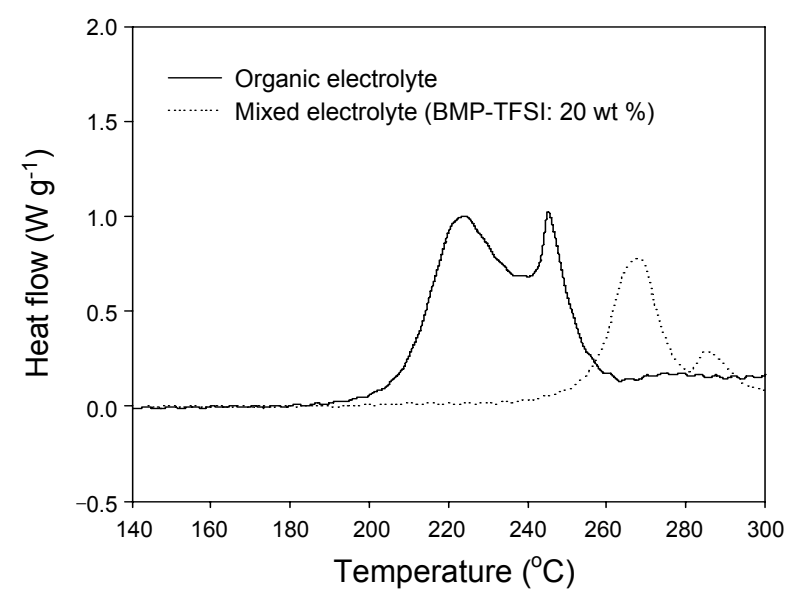

Figure 6. DSC profiles of $\mathrm{Li}_{1-\mathrm{x}} \mathrm{CoO}_{2}$ cathode materials charged to $4.2 \mathrm{~V}$ in the organic electrolyte and the mixed electrolytes containing 20 wt \% BMP-TFSI.

tical ac impedance spectra, except for a small difference in the electrolyte resistance. This result indicates that the presence of BMP-TFSI in the mixed electrolyte has little effect on the interfacial resistance in the pristine cell. After 100 charge and discharge cycles, two overlapped semicircles are found. According to previous impedance analysis studies, ${ }^{24-26}$ the semicircle in the high frequency range can be attributed to resistance due to $\mathrm{Li}^{+}$ion migration through the electrode surface SEI film, while the semicircle in the medium-to-low frequency range is due to the charge transfer resistance between the electrode and electrolyte. It is found that both the surface film resistance and the charge transfer resistance increase as the BMP-TFSI content increases from 0 to $30 \mathrm{wt} \%$. This result is associated with the growth of the resistive layer on the electrode surface due to the progressive decomposition of BMP-TFSI. The resistive layer also hampers charge transport at the electrode and electrolyte interface, which causes an increase in charge transfer resistance. Also an additional contribution from a loss of interfacial contact between the electrode materials and the electrolyte solution, arising from the highly viscous nature of IL, cannot be excluded. Thus, proper control of the BMP-TFSI content is very impor- tant for achieving good capacity retention and stable interfacial behavior.

In order to investigate the flammable behavior of the mixed electrolyte containing BMP-TFSI, a flammability test was performed. As clearly shown in previous work, ${ }^{12}$ the flammability of a given electrolyte solution is known to be proportional to the SET value. Figure 5 shows the normalized SET values associated with mixed electrolytes having different BMP-TFSI contents. It is clearly observed that the flammability of the mixed electrolyte decreases with increasing BMP-TFSI content. We also confirmed that BMP-TFSI was non-flammable. Figure 6 compares the DSC profiles of the charged cathode materials in the organic electrolyte and the mixed electrolyte containing $20 \mathrm{wt} \%$ BMP-TFSI. The trace related to the organic electrolyte has two exothermic peaks occurring between 190 and $260^{\circ} \mathrm{C}$, which are associated with the reaction of the organic solvents with the oxygen released from the charge $\mathrm{Li}_{1-x} \mathrm{CoO}_{2}$ lattice. ${ }^{8,26,27}$ The heat generated by the thermal reaction of the charged cathode with the electrolyte is reduced, and the onset temperature increases by about $50{ }^{\circ} \mathrm{C}$, when passing to the mixed electrolyte. This result confirms the beneficial role of the BMP-TFSI in enhancing the thermal stability of the charged cathode material. Accordingly, the results of the SET and DSC tests concur to demonstrate the use of IL-organic solution mixed electrolytes improves the safety level of lithium-ion batteries.

\section{Conclusions}

Mixed electrolytes containing non-flammable BMP-TFSI were prepared and investigated. The cathodic stability of the electrolyte could be extended to $0 \mathrm{~V}$ via the formation of a protective film due to the reductive decomposition of organic solvents on the electrode surface, which is necessary for applications in lithium-ion batteries with graphitized carbon anodes. The lithium-ion cells composed of a carbon anode and a $\mathrm{LiCoO}_{2}$ cathode exhibited reversible charge and discharge cycling in these mixed electrolytes. Proper control of the BMP-TFSI content was proven to be very important for achieving good capacity retention and stable interfacial behavior. Flammability tests and DSC studies showed that the safety of the cells could be improved by using the mixed electrolytes.

Acknowledgments. This work was supported by the IT R\&D program of MKE/KEIT [KI002176-2010-02, Development of 3.6Ah Class Cylindrical Type Lithium Secondary Battery].

\section{References}

1. Welton, T. Chem. Rev. 1999, 99, 2071

2. Armand, M.; Endres, F.; MacFarlane, D. R.; Ohno, H.; Scrosati, B. Nature Mater. 2009, 9, 621 .

3. Lewandowski, A.; Swiderska-Mocek, A. J. Power Sources 2009, 194, 601.

4. Fernicola, A.; Croce, F.; Scrosati, B.; Watanabe, T.; Ohno, H. J. Power Sources 2007, 174, 342.

5. Reale, P.; Fernicola, A.; Scrosati, B. J. Power Sources 2009, 194, 182.

6. Yim, T.; Lee, H. Y.; Kim, H. J.; Mun, J.; Kim, S.; Oh, S. M.; Kim, Y. G. Bull. Korean Chem. Soc. 2007, 28, 1567.

7. Shin, J. H.; Cairns, E. J. J. Power Sources 2008, 177, 537. 
8. Larush, L.; Borgel, V.; Markevich, E.; Haik, O.; Zinigrad, E.; Aurbach, D. J. Power Sources 2009, 189, 217.

9. Borgel, V.; Markevich, E.; Aurbach, D.; Semrau, G.; Schmidt, M. J. Power Sources 2009, 189, 331.

10. Hassoun, J.; Fernicola, A.; Navarra, M. A.; Panero, S.; Scrosati, B. J. Power Sources 2010, 195, 574.

11. Eo, S. M.; Cha, E.; Kim, D. W. J. Power Sources 2009, 189, 766.

12. Xu, K.; Ding, M. S.; Zhang, S.; Allen, J. L.; Jow, T. R. J. Electrochem. Soc. 2002, 149, A622.

13. Sivakkumar, S. R.; MacFarlane, D. R.; Forsyth, M.; Kim, D. W.J. Electrochem. Soc. 2007, 154, A834.

14. Aurbach, D.; Ein-Eli, Y.; Markovsky, B.; Zaban, Z. J. Electrochem. Soc. 1995, 142, 885 .

15. Ein-Eli, Y.; Thomas, S. R.; Chadha, R.; Blakley, T. J.; Koch, V. R. J. Electrochem. Soc. 1997, 144, 823.

16. Mogi, R.; Inaba, M.; Jeong. S.-K.; Iriyama, Y.; Abe, T.; Ogumi, Z. J. Electrochem. Soc. 2002, 149, A1578.

17. Sato, T; Maruo, M.; Marukane, S.; Takagi, K. J. Power Sources
2004, 138, 253.

18. Ota, H; Sakata, Y; Otake, Y; Shima, K.; Ue, M.; Yamaki, J. J. Electrochem. Soc. 2005, 151, A1778.

19. Zhang, S. S. J. Power Sources 2006, 162, 1379.

20. El Ouatani, L.; Dedryvere, R.; Siret, C.; Biensan, P.; Gonbeau, D. J. Electrochem. Soc. 2009, 156, A468.

21. Holzapfel, M.; Jost, C.; Prodi-Schwab, A.; Krumeich, F.; Wursig, A.; Buqa, H.; Novak, P. Carbon 2005, 43, 1488.

22. Katayama, Y.; Yukumoto, M.; Miuta, T. Electrochem. Solid State Lett. 2003, 6, A96.

23. Zheng, H.; Jiang, K.; Abe, T.; Ogumi, Z. Carbon 2006, 44, 203.

24. Funabiki, A.; Inaba, M.; Ogumi, Z. J. Power Sources 1997, 68, 227.

25. Levi, M. D.; Salitra, G.; Markovsky, B.; Teller, H.; Aurbach, D.; Heider, U.; Heider, L. J. Electrochem. Soc. 1999, 146, 1279.

26. Baba, Y.; Okada, S.; Yamaki, J. Solid State Ionics 2002, 148, 311.

27. Sakabe, H.; Matsumoto, H.; Tatsumi, K. Electrochim. Acta 2007, 53,1048 . 